\title{
二甲梄和乙醇低压层流预混火焰的对比研究
}

\author{
高 健 ${ }^{1,3}$ 赵黛青 ${ }^{1, *}$ 汪小懸 ${ }^{1}$ 蒋利桥 1

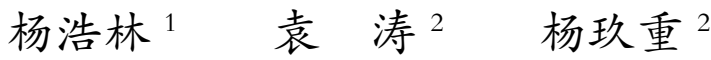 \\ ( ${ }^{1}$ 中国科学院广州能源研究所, 广州 $510640 ;{ }^{2}$ 中国科学技术大学国家同步辐射实验室, 合肥 230029 ; \\ 3 中国科学院研究生院, 北京 100049)
}

\begin{abstract}
摘要：利用分子束质谱结合真空紫外同步辐射光电离技术对相同燃烧条件下的低压层流预混二甲醚/氧气/氩 气和乙醇/氧气/氩气火焰进行研究. 通过测量光电离效率曲线, 识别了二甲醚和乙醇火焰的中间物种, 得到相应 的火焰质谱; 通过测量火焰中各物种在燃烧炉炉膛各位置的电离信号强度, 得到了各物种的摩尔分数分布曲线. 结合两种燃料分子不同的化学结构及详细的燃烧化学反应机理, 分析了两火焰中间物种生成特性的异同. 研究 结果表明: 甲醛为两火焰中最主要的 $\mathrm{C}_{1}$ 中间物种; 二甲醚火焰趋向于生成 $\mathrm{C}_{1}$ 中间物种, $\mathrm{C}_{2}$ 物种摩尔分数较低; 乙醇火焰中乙醛、乙烯、乙炔和乙烯酮等 $\mathrm{C}_{2}$ 中间物种的摩尔分数明显高于二甲醚火焰中的值.
\end{abstract}

关键词：同步辐射；单光子电离；二甲醚；乙醇；层流预混火焰

中图分类号: $\mathrm{O} 643$

\section{Comparative Study of Dimethyl Ether and Ethanol Premixed Laminar Flames at Low Pressure}

\author{
GAO Jian $^{1,3} \quad$ ZHAO Dai-Qing ${ }^{1, *} \quad$ WANG Xiao-Han $^{1} \quad$ JIANG Li-Qiao $^{1}$ \\ YANG Hao-Lin $^{1} \quad$ YUAN Tao ${ }^{2} \quad$ YANG Jiu-Zhong ${ }^{2}$ \\ ( ${ }^{1}$ Guangzhou Institute of Energy Conversion, Chinese Academy of Sciences, Guangzhou $\quad 510640$, P. R. China; \\ ${ }^{2}$ National Synchrotron Radiation Laboratory, University of Science and Technology of China, Hefei 230029, P. R. China; \\ ${ }^{3}$ Graduate University of Chinese Academy of Sciences, Beijing 100049, P. R. China)
}

\begin{abstract}
Low pressure premixed laminar dimethyl ether (DME)/oxygen/argon and ethanol/oxygen/argon flames (equivalence ratio: 1.0) were studied by molecular-beam sampling mass spectrometry (MBMS) combined with the tunable synchrotron radiation photoionization technique. Combustion intermediates were identified by measuring photoionization efficiency (PIE) spectra, and the mole fraction profiles of these species at different flame positions were presented. Similarities and differences of main intermediate formation characteristics between the two flames were analyzed based on derived reaction mechanisms. Experimental results show that both flames contain the same intermediates such as $\mathrm{CH}_{3}, \mathrm{CH}_{4}, \mathrm{C}_{2} \mathrm{H}_{2}, \mathrm{C}_{2} \mathrm{H}_{4}, \mathrm{CH}_{2} \mathrm{O}, \mathrm{CH}_{3} \mathrm{OH}, \mathrm{CH}_{2}=\mathrm{C}=\mathrm{O}, \mathrm{CH}_{3} \mathrm{CHO}$, and $\mathrm{CH}_{2} \mathrm{CHOH}$. In the DME flame, the concentration of the $\mathrm{C}_{1}$ species is much higher than that of the $\mathrm{C}_{2}$ species, i.e., $\mathrm{C}_{1}$ intermediates tend to form rather than $\mathrm{C}_{2}$ intermediates in a DME flame. In addition, formaldehyde is the dominant $\mathrm{C}_{1}$ species in each flame. The concentrations of $\mathrm{C}_{2}$ species like ethylene, acetaldehyde, acetylene, and ketene in the ethanol flame are remarkably higher than that found in the DME flame.
\end{abstract}

Key Words : Synchrotron radiation; Single-photon ionization; Dimethyl ether; Ethanol;

Premixed laminar flame

Received: July 14, 2009; Revised: September 22, 2009; Published on Web: November 3, 2009.

*Corresponding author. Email: zhaodq@ms.giec.ac.cn; Tel: +86-20-87057765.

The project was supported by the National Natural Science Foundation of China (50806079) and Natural Science Foundation of Guangdong Province, China (8151007006000014).

国家自然科学青年基金(50806079)和广东省自然科学基金(8151007006000014)资助项目

C Editorial office of Acta Physico-Chimica Sinica 
随着石油资源的日益短缺和排放法规的日益严 格, 替代燃料燃烧特性的研究已经成为当今的一个 热点.二甲醚和乙醇均可由煤、生物质等燃料通过热 化学方法制取, 来源广泛且产生的常规污染物排放 低, 且能够与现有大部分油品掺混使用. 在工业领 域, 可替代或部分替代柴油、汽油等动力燃料, 且对 发动机等然烧设备的改动要求不高; 在民用领域, 也可作为液化石油气等民用燃料的替代品使用. 因 此, 两者均被认为是具较大发展潜力的含氧替代燃 料 $[1,2]$.

二甲醚 $\left(\mathrm{CH}_{3} \mathrm{OCH}_{3}\right)$ 沸点为 $-24.9{ }^{\circ} \mathrm{C}$, 常温常压下 是一种无色有轻微醚香味的气体, 密度 $0.66 \mathrm{~g} \cdot \mathrm{mL}^{-1}$ $\left(20{ }^{\circ} \mathrm{C}\right)$, 与乙醇 $\left(\mathrm{C}_{2} \mathrm{H}_{5} \mathrm{OH}\right)$ 互为同分异构体, 相对分子 质量同为 46 , 含氧 $34.8 \%$, 但二甲醚分子结构中没 有 $\mathrm{C}-\mathrm{C}$ 键, 只有 $\mathrm{C}-\mathrm{H}$ 和 $\mathrm{C}-\mathrm{O}$ 键, 其低位热值为 $27.60 \mathrm{MJ} \cdot \mathrm{kg}^{-1}$, 略高于乙醇 $\left(26.75 \mathrm{MJ} \cdot \mathrm{kg}^{-1}\right)^{[2]}$.

当前对二甲醚和乙醇燃烧的化学反应机理的研 究已经深人开展, 同步辐射真空紫外光电离结合分 子束质谱技术作为燃烧诊断的一种新方法, 因其能 够探测各种燃烧中间体和重要自由基, 为二甲醚和 乙醇燃烧动力学反应机理研究的发展提供了有价值 的实验数据: Cool 等 ${ }^{[3]}$ 对富燃料的二甲醚低压层流 预混火焰进行了实验研究, 并与其之前修正的二甲 醚高温氧化机理 ${ }^{[4]}$ 进行了对比, 实验结果与反应动 力学模型符合较好; Kasper 等 ${ }^{[5,6]}$ 分别利用电子轰击 电离和同步辐射单光子电离结合分子束质谱技术对 乙醇及乙醇/丙烯的低压层流预混平面火焰进行了 实验研究, 比较认为两套装置的实验结果总体上一 致, 苯的生成随乙醇掺混量的增大而减少, 为芳香烃 及醛类物种生成路径的研究提供了重要的实验数 据; Wang 等 ${ }^{[7]}$ 同样利用电子轰击和单光子电离结合 分子束质谱技术对二甲醚/丙烯和乙醇/丙烯火焰进 行了实验研究, 结合相关化学反应机理 ${ }^{[6,8-10]}$ 的重要 反应路径对实验进行了指导和分析, 结果表明, 二甲 醚和乙醇的掺混降低了火焰中苯的浓度, 二甲醚掺 混使得火焰中的甲醛浓度明显增大. 目前国内较多 集中于对两种燃料及其与汽油、柴油掺混后的内燃 机燃烧特性的研究 ${ }^{[11-16]}$.

当前, 对由于两者分子结构的不同导致的燃烧 路径、中间产物的差异性分析尚未见系统报道, 本文 利用分子束质谱结合真空紫外同步辐射光电离技术 对两种燃料的燃烧特性尤其是火焰中间物种的生成 特性进行深人的对比研究, 并结合前人相同或相近
实验条件下的重要实验结论 ${ }^{[3,7]}$ 及适用的二甲醚和乙 醇的氧化反应机理 ${ }^{[4,6,8-10,17-19]}$, 对其中的部分实验结果 进行了分析. 定量分析的结果对于掌握含氧燃料的 燃烧机理, 掌握醛酮等潜在污染物的排放特性, 完善 和优化燃烧技术均具有重要的参考价值.

\section{1 实验装置}

实验在中国科学技术大学国家同步辐射实验室 的燃烧实验站进行. 实验装置主要由燃烧室、差分 室、电离室和反射式飞行时间质谱仪组成, 详细介绍 参见文献[20]. 燃料、氧气、氩气流量通过质量流量 控制器精确控制, 混合后进人一直径 $60 \mathrm{~mm}$ 燃烧炉 形成圆盘状的层流预混火焰. 通过计算机控制步进 马达实现燃烧器在火焰传播方向上的移动, 这样就 可以改变火焰与取样喷嘴的相对位置, 从而探测火 焰中物种的空间摩尔分布. 实验中, 燃烧室压力通过 节流阀来调节, 燃烧室压力稳定在 $4.0 \mathrm{kPa}$.

首先, 定义燃空比 $\phi=\left(n_{\mathrm{f}} / n_{\mathrm{a}}\right) /\left(n_{\mathrm{f}} / n_{\mathrm{a}}\right)_{\mathrm{st}}$, 其中, $n_{\mathrm{f}}$ 和 $n_{\mathrm{a}}$ 分别表示燃料和空气的物质的量, 下标 st 表示等 化学计量. 进口燃空比控制为 1.0, 燃料(分别为二甲 醚和乙醇) 、氧气和氩气流量分别为 $0.429 、 1.286$ 和 $1.286 \mathrm{~L} \cdot \mathrm{min}^{-1}$. 实验主要分为两个部分: 一是固定燃 烧炉的位置, 扫描光子能量, 从而得到不同质荷比的 离子信号强度随光子能量的变化曲线, 即光电离效 率谱(PIE), 通过光电离效率谱得到分子的电离能, 与文献中电离能相比较以确定物种成分; 二是固定 光子能量, 扫描燃烧炉位置, 得到火焰中各物种成分 的空间摩尔分数分布曲线. 实验中测得火焰中各物 种的光电离信号强度, 应用文献[21]的方法根据光 电离信号强度计算出各物种在各位置的摩尔分数.

\section{2 实验结果及分析}

\section{1 火焰光电离质谱比较}

图 $1 、 2$ 分别为在燃烧室压力 $4.0 \mathrm{kPa}$ 下的二甲 醚、乙醇火焰质谱图, 取样位置距离燃烧器表面 2.0 $\mathrm{mm}$, 光子能量为 $11.80 \mathrm{eV}$. 质谱图中每一信号强度 峰值对应一种质荷比的燃烧产物, 通过扫描光子能 量得到每个燃烧产物的光电离效率谱, 结合文献中 物种电离能值可确定每种产物并区分同分异构体, 具体方法参见文献[22]. 以上火焰质谱图中质荷比为 $15 、 26 、 28 、 30 、 31 、 32 、 42 、 44 、 46 、 60$ 的电离信号分别 对应甲基 $\left(\mathrm{CH}_{3}\right)$ 、乙炔 $\left(\mathrm{C}_{2} \mathrm{H}_{2}\right)$ 、乙烯 $\left(\mathrm{C}_{2} \mathrm{H}_{4}\right)$ 、甲醛 $\left(\mathrm{CH}_{2} \mathrm{O}\right)$ 、 甲氧基 $\left(\mathrm{CH}_{3} \mathrm{O}\right)$ 、甲醇 $\left(\mathrm{CH}_{3} \mathrm{OH}\right)$ 、乙烯酩 $\left(\mathrm{H}_{2} \mathrm{C}=\mathrm{C}=\mathrm{O}\right)$ 、 
乙醛 $\left(\mathrm{CH}_{3} \mathrm{CHO}\right)$ 和乙烯醇 $\left(\mathrm{CH}_{2}=\mathrm{CHOH}\right)$ 、二甲醚 $\left(\mathrm{CH}_{3} \mathrm{OCH}_{3}\right)$ 或乙醇 $\left(\mathrm{C}_{2} \mathrm{H}_{5} \mathrm{OH}\right)$ 、甲基乙基醚 $\left(\mathrm{CH}_{3} \mathrm{OC}_{2} \mathrm{H}_{5}\right)$. 乙醛和乙烯醇质荷比同为 44 , 且电离能分别为 10.23 和 $9.62 \mathrm{eV}$, 在 $11.80 \mathrm{eV}$ 的光子能量下均被电离, 因 此这里对应于质荷比 44 的信号强度事实上是两种 同分异构体的电离信号强度之和. 虽然甲醇和氧气 质荷比同为 32 , 但由于氧气的电离能为 $12.07 \mathrm{eV}$, 氧气在 $11.80 \mathrm{eV}$ 的光子能量下并未发生电离, 因此 对应于质荷比 32 的信号强度仅由甲醇电离产生.

对比二甲醚和乙醇火焰质谱, 在二甲醚火焰中 探测到甲基乙基醚信号, 乙醇火焰中并未出现; 乙 醇火焰中出现甲氧基信号, 而在二甲醚火焰中, 甲 氧基信号极弱. 这里需要特别指出, 由于不同实验 工况下光强不同且不同物种光电离截面不同, 因此, 信号的强弱并不能直接说明物质的量的多少, 不同 物种的定量分析将通过下文的摩尔分数曲线进行 详细分析.

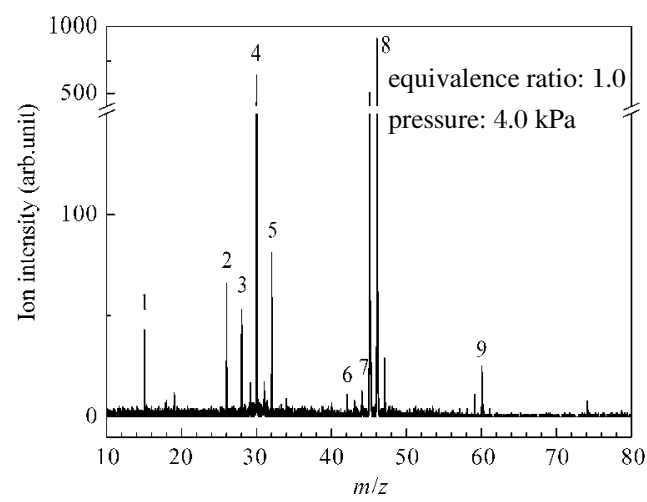

图 1 二甲醚火焰质谱图

Fig.1 Mass spectrum of the DME flame

(1) $\mathrm{CH}_{3}$, (2) $\mathrm{C}_{2} \mathrm{H}_{2}$, (3) $\mathrm{C}_{2} \mathrm{H}_{4}$, (4) $\mathrm{CH}_{2} \mathrm{O}$, (5) $\mathrm{CH}_{3} \mathrm{OH}$, (6) $\mathrm{CH}_{2}=\mathrm{C}=\mathrm{O}$,

(7) $\mathrm{CH}_{3} \mathrm{CHO}$ and $\mathrm{CH}_{2}=\mathrm{CHOH}$, (8) $\mathrm{CH}_{3} \mathrm{OCH}_{3}$, (9) $\mathrm{CH}_{3} \mathrm{OC}_{2} \mathrm{H}_{5}$

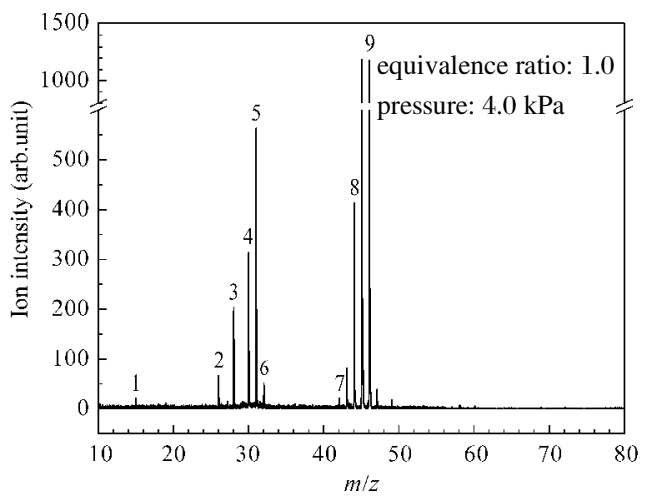

图 2 乙醇火焰质谱图

Fig.2 Mass spectrum of the ethanol flame (1) $\mathrm{CH}_{3}$, (2) $\mathrm{C}_{2} \mathrm{H}_{2}$, (3) $\mathrm{C}_{2} \mathrm{H}_{4}$, (4) $\mathrm{CH}_{2} \mathrm{O}$, (5) $\mathrm{CH}_{3} \mathrm{O}$, (6) $\mathrm{CH}_{3} \mathrm{OH}$, (7) $\mathrm{CH}_{2}=\mathrm{C}=\mathrm{O}$, (8) $\mathrm{CH}_{3} \mathrm{CHO}$ and $\mathrm{CH}_{2}=\mathrm{CHOH}$, (9) $\mathrm{C}_{2} \mathrm{H}_{5} \mathrm{OH}$

\section{2 火焰中的主要物种摩尔分数分布比较}

图 3、4 分别为二甲醚、乙醇火焰主要产物摩尔 分数曲线. 燃料(二甲醚或乙醇)在距离燃烧炉表面 3 $\mathrm{mm}$ 内已经消耗完全. 比较两火焰中 $\mathrm{CO}$ 和 $\mathrm{CO}_{2}$ 的 生成情况, 相对于二甲醚, 乙醇的燃烧生成了较多的 $\mathrm{CO}_{2}$, 较少的 $\mathrm{CO}$.

二甲醚火焰中水的摩尔分数在距离燃烧炉表 面 $5.5 \mathrm{~mm}$ 处出现峰值, 之后下降直到稳定值 0.36 , 而对于乙醇, 这一峰值并不明显, 但最终的稳定值几 乎与二甲醚相等, 同为 0.36 . 两火焰中有少量的氢气 产生, 相对于乙醇火焰, 二甲醚火焰中产生氢气的摩 尔分数峰值更为明显, 这可能是因为二甲醚分子中 所有的 $\mathrm{H}$ 原子通过 $\mathrm{C}-\mathrm{H}$ 键连接在 $\mathrm{C}$ 原子上, 而乙 醇分子中其中一个 $\mathrm{H}$ 原子通过 $\mathrm{O}-\mathrm{H}$ 键连接在 $\mathrm{O}$ 原子上, 由于 $\mathrm{O}-\mathrm{H}$ 键平均键能高于 $\mathrm{C}-\mathrm{H}$ 键, 二甲 醚分子的脱氢反应更易发生造成其氢气生成量略多 于乙醇.

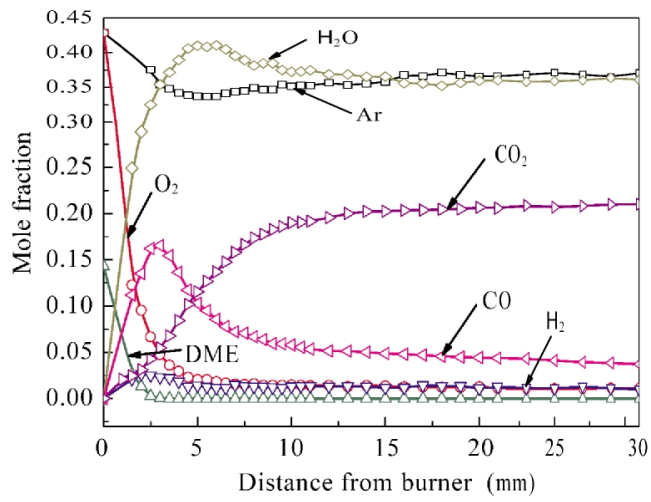

图 3 二甲醚火焰主要物种摩尔分数曲线

Fig.3 Mole fraction profiles for the major species of the DME flame

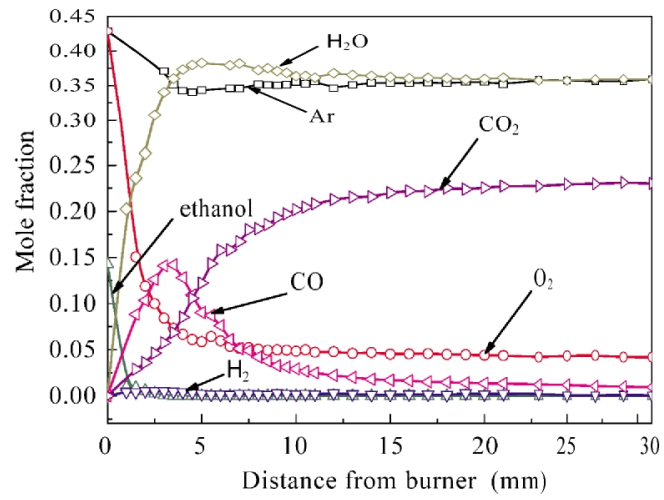

图 4 乙醇火焰主要物种摩尔分数曲线

Fig.4 Mole fraction profiles for the major species of the ethanol flame 


\section{3 火焰中的中间物种摩尔分数分布比较}

图 5、6 分别为二甲醚、乙醇火焰中主要 $\mathrm{C}_{1}$ 中间 物种的摩尔分数分布曲线, 包括甲基、甲烷、甲醇和 甲醛四种物质. 两火焰中, 甲醛为最主要的 $\mathrm{C}_{1}$ 中间 物种, 其摩尔分数明显高于其它 $\mathrm{C}_{1}$ 物种. 二甲醚和 乙醇火焰中甲醛的生成机理基本一致 ${ }^{[3,4,7,7,10,17]}$, 主要 经过以下反应路径生成, 其中含有 “ $\mathrm{M}$ ” 的基元反应 为三体(3-rd body)反应:

\section{$\mathrm{CH}_{3} \mathrm{OCH}_{3}\left(\mathrm{CH}_{3} \mathrm{CH}_{2} \mathrm{OH}\right)+\mathrm{R} \rightarrow$ \\ 和

$$
\mathrm{CH}_{3}+\mathrm{HO}_{2} \rightarrow \mathrm{CH}_{3} \mathrm{O}+\mathrm{OH}
$$$$
\mathrm{CH}_{3} \mathrm{O}(+\mathrm{M}) \rightarrow \mathrm{CH}_{2} \mathrm{O}+\mathrm{H}(+\mathrm{M})
$$

$\mathrm{CH}_{3} \mathrm{OCH}_{2}\left(\mathrm{CH}_{3} \mathrm{CH}_{2} \mathrm{O}\right)+\mathrm{RH} \quad\left(\mathrm{R}=\mathrm{O}_{2}, \mathrm{H}, \mathrm{OH}, \mathrm{CH}_{3}\right)$ $\mathrm{CH}_{3} \mathrm{OCH}_{2}\left(\mathrm{CH}_{3} \mathrm{CH}_{2} \mathrm{O}\right) \rightarrow \mathrm{CH}_{3}+\mathrm{CH}_{2} \mathrm{O}$

可见, 两种火焰中的甲醛生成基本都是通过(i) 燃料脱氢 $\rightarrow \beta$ 裂解 $\rightarrow$ 甲醛及(ii)甲基加氧 $\rightarrow$ 甲氧基 脱氢 $\rightarrow$ 甲醛两个过程, 另外, 甲醛的消耗机理在两火 焰中也基本相同 $33,4,7,9,10,17]$ :

\section{$\mathrm{CH}_{2} \mathrm{O}+\mathrm{R} \rightarrow \mathrm{HCO}+\mathrm{RH} \quad\left(\mathrm{R}=\mathrm{O}_{2}, \mathrm{H}, \mathrm{OH}, \mathrm{CH}_{3}\right)$ \\ $\mathrm{HCO}+\mathrm{R} \rightarrow \mathrm{CO}+\mathrm{RH} \quad\left(\mathrm{R}=\mathrm{O}_{2}, \mathrm{H}, \mathrm{OH}, \mathrm{CH}_{3}\right)$}

因此, 如图 5、6 中所示, 两火焰中甲醛的 $0 \mathrm{~mm}$ 取样位置摩尔分数和摩尔分数峰值及其出现位置都 比较接近, 但乙醇火焰中甲醛完全耗尽的位置略滞 后于二甲醚, 二甲醚火焰中甲醛的消耗更为迅速.

甲烷通过甲基夺取其他分子中的 $\mathrm{H}$ 原子生成, 两火焰中甲烷摩尔分数曲线的上升阶段皆较为平 缓, 二甲醚火焰中甲基、甲烷生成量高于乙醇火焰. 在乙醇火焰中, 探测到了甲醇摩尔分数先上升后下 降直至完全耗尽的过程; 而在二甲醚火焰中, 在取 样位置 $0 \mathrm{~mm}$ 处甲醇的摩尔分数已经达到峰值, 随

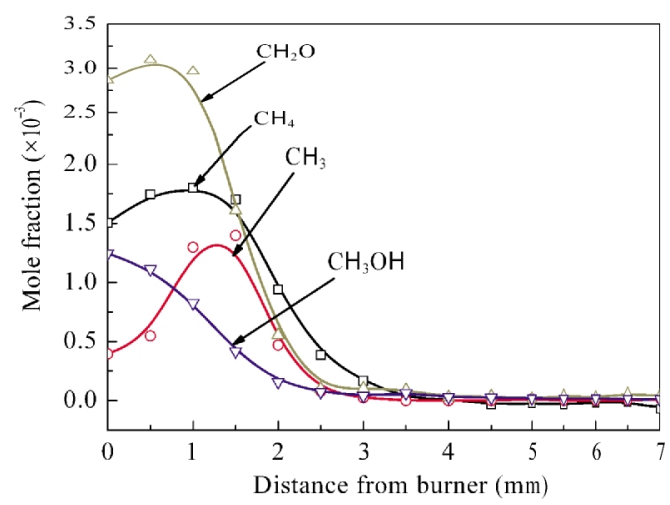

图 5 二甲醚火焰 $\mathbf{C}_{1}$ 物种摩尔分数曲线

Fig.5 Mole fraction profiles for the $C_{1}$ species of the DME flame

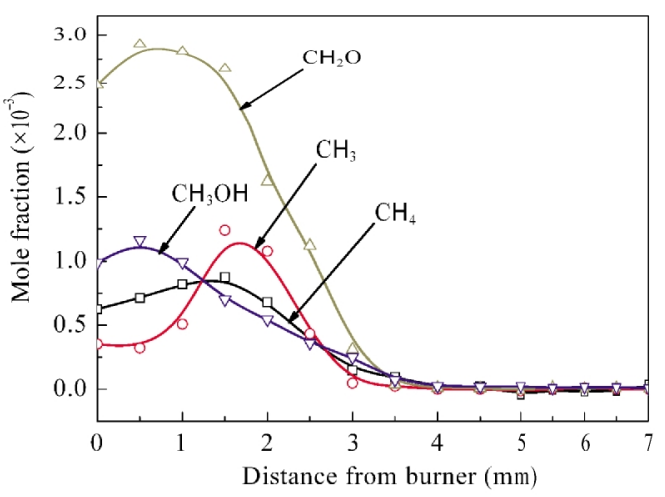

图 6 乙醇火焰 $\mathbf{C}_{1}$ 物种摩尔分数曲线

Fig.6 Mole fraction profiles for the $\mathrm{C}_{1}$ species of the ethanol flame

后下降直到耗尽, 这说明, 乙醇火焰中甲醇生成反应 的发生略滞后于二甲醚火焰.

二甲醚火焰 $\mathrm{C}_{1}$ 中间物种在 $3.0 \mathrm{~mm}$ 处几乎完全 耗尽, 而乙醇火焰 $\mathrm{C}_{1}$ 物种耗尽位置在 $4.0 \mathrm{~mm}$ 处, 略 滞后于二甲醚火焰, 乙醇火焰反应区域略宽于二甲 醚火焰. 总的说来, 二甲醚主要 $\mathrm{C}_{1}$ 物种摩尔分数曲 线峰值出现位置较靠近燃烧炉, 且峰值略高于乙醇 火焰, 这可能是因为相对于乙醇分子的 $\mathrm{C}-\mathrm{C}$ 键, 二 甲醚分子的 $\mathrm{C}-\mathrm{O}$ 键更易断裂, 形成 $\mathrm{C}_{1}$ 中间物种较 为容易。

图 7、8 分别为二甲醚、乙醇火焰中主要 $\mathrm{C}_{2}$ 中间 物种摩尔分数分布, 主要包括乙炔、乙烯、乙醛和乙 烯酮. 乙醇火焰中的 $\mathrm{C}_{2}$ 物种摩尔分数峰值分别为 $7.0 \times 10^{-3}\left(\mathrm{CH}_{3} \mathrm{CHO}\right) 、 4.8 \times 10^{-3}\left(\mathrm{C}_{2} \mathrm{H}_{4}\right) 、 1.9 \times 10^{-3}\left(\mathrm{CH}_{2} \mathrm{CO}\right) 、$ $0.7 \times 10^{-3}\left(\mathrm{C}_{2} \mathrm{H}_{2}\right)$, 其中乙醛为生成量最高的 $\mathrm{C}_{2}$ 中间物 种; 二甲醚火焰中生成量最高的 $\mathrm{C}_{2}$ 中间物种为 $\mathrm{C}_{2} \mathrm{H}_{4}$, 其摩尔分数峰值仅为 $0.96 \times 10^{-3}$, 可见乙醇火焰 中各 $\mathrm{C}_{2}$ 中间物种的生成量明显高于二甲醚火焰.

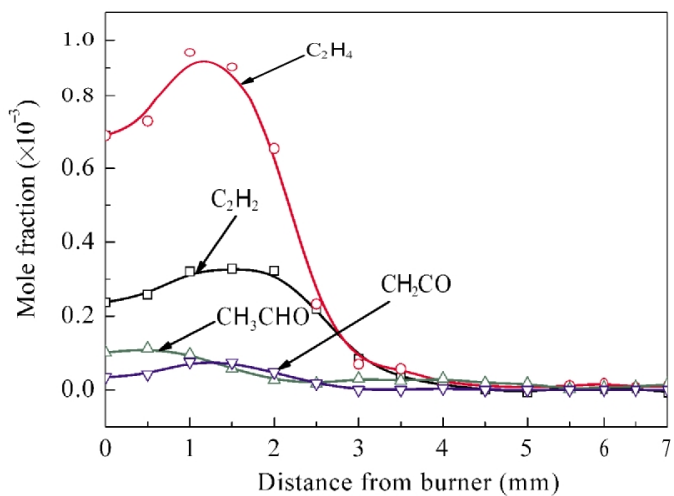

图 7 二甲醚火焰 $\mathbf{C}_{2}$ 物种摩尔分数曲线

Fig.7 Mole fraction profiles for the $C_{2}$ species of the DME flame 


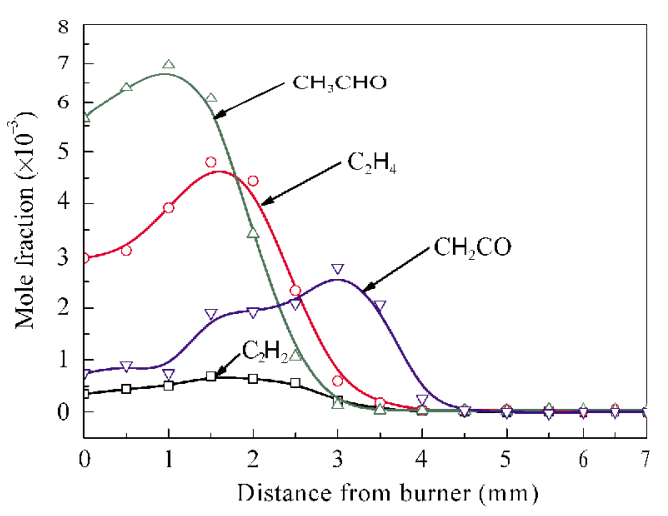

图 8 乙醇火焰 $\mathbf{C}_{2}$ 物种摩尔分数曲线

Fig.8 Mole fraction profiles for the $C_{2}$ species of the ethanol flame

在二甲醚火焰中, 乙烯由甲基的复合反应生 成 ${ }^{[3,4,17]}$.

$\mathrm{CH}_{3}+\mathrm{CH}_{3} \rightarrow \mathrm{C}_{2} \mathrm{H}_{5}+\mathrm{H}$

$\mathrm{C}_{2} \mathrm{H}_{5}+\mathrm{R} \rightarrow \mathrm{C}_{2} \mathrm{H}_{4}+\mathrm{RH} \quad\left(\mathrm{R}=\mathrm{H}, \mathrm{O}_{2}\right)$

甲基是一种极其活泼的基团, 参与众多基元反 应, 二甲醚火焰中乙烯的生成依赖于甲基的复合, 决 定了乙烯的生成量必然极少; 而在乙醇火焰中, 乙 烯主要通过两条路径生成 ${ }^{[7,9,10]}$ :

\section{$\mathrm{C}_{2} \mathrm{H}_{5} \mathrm{OH}+\mathrm{OH} \rightarrow \mathrm{C}_{2} \mathrm{H}_{4} \mathrm{OH}+\mathrm{H}_{2} \mathrm{O}$ \\ $\mathrm{C}_{2} \mathrm{H}_{4} \mathrm{OH} \rightarrow \mathrm{C}_{2} \mathrm{H}_{4}+\mathrm{OH}$}

和

$\mathrm{C}_{2} \mathrm{H}_{5} \mathrm{OH}(+\mathrm{M}) \rightarrow \mathrm{C}_{2} \mathrm{H}_{4}+\mathrm{H}_{2} \mathrm{O}(+\mathrm{M})$

乙醇分子经脱氢+脱羟基的过程即可生产乙烯, 因此, 乙醇火焰中乙烯摩尔分数明显高于二甲醚火 焰.

两火焰中乙炔的生成路径基本相同: 乙烯与羟 基等反应脱氢形成乙烯基, 乙烯基继续脱去氢原子 即生成乙炔 ${ }^{[9,17}$. 相对来讲, 乙炔比较稳定, 因此其摩 尔分数分布曲线上升和下降较为平缓. 乙炔的生成 几乎全部来源于乙烯, 因此其摩尔分数低于乙烯的, 且峰值出现位置滞后于乙烯.

乙醛是乙醇火焰中最重要的 $\mathrm{C}_{2}$ 中间物种, 乙醇 分子在 $\mathrm{H}$ 或 $\mathrm{OH}$ 的作用下脱氢生成乙烯醇, 再在氧 气的氧化作用下脱去羟基氢生成乙醛 ${ }^{[7,9,10]}$ :

$\mathrm{C}_{2} \mathrm{H}_{5} \mathrm{OH}+\mathrm{R} \rightarrow \mathrm{CH}_{3} \mathrm{CHOH}+\mathrm{RH} \quad(\mathrm{R}=\mathrm{H}, \mathrm{OH})$

$\mathrm{CH}_{3} \mathrm{CHOH}+\mathrm{O}_{2} \rightarrow \mathrm{CH}_{3} \mathrm{CHO}+\mathrm{HO}_{2}$

另有少量乙醛会通过先脱羟基氢生成:

$\mathrm{C}_{2} \mathrm{H}_{5} \mathrm{OH}+\mathrm{OH} \rightarrow \mathrm{CH}_{3} \mathrm{CH}_{2} \mathrm{O}+\mathrm{H}_{2} \mathrm{O}$

$\mathrm{CH}_{3} \mathrm{CH}_{2} \mathrm{O}+\mathrm{M} \rightarrow \mathrm{CH}_{3} \mathrm{CHO}+\mathrm{H}+\mathrm{M}$
乙醇燃烧的乙醛生成特性决定了乙醛为乙醇火 焰中摩尔分数最高的中间物种, 且摩尔分数峰值位 置最为接近燃烧炉表面. 而在二甲醚火焰中, 我们仅 探测到极少量的乙醛, 其摩尔分数峰值仅为 $1.0 \times 10^{-4}$. 另外, 二甲醚火焰中乙烯酮摩尔分数较低, 峰值仅为 $7.5 \times 10^{-5}$.

结合两种燃料分子化学结构, 二甲醚本身并不 含有 $\mathrm{C}-\mathrm{C}$ 键, 生成一个含有 $\mathrm{C}-\mathrm{C}$ 键的 $\mathrm{C}_{2}$ 中间产 物必定要经过碳氧键断裂 $\rightarrow \mathrm{C}-\mathrm{C}$ 键聚合的过程, 而这在燃空比为 1.0 的预混火焰中并不容易发生, 因此, 二甲醚 $\mathrm{C}_{2}$ 中间产物摩尔分数远低于乙醇.

\section{3 结 论}

通过测试燃空比均为 1.0 的二甲醚和乙醇低压 层流预混火焰质谱、光电离效率曲线及摩尔分数分 布曲线, 分析了两火焰中主要 $\mathrm{C}_{1} 、 \mathrm{C}_{2}$ 中间物种的生 成特性的异同.

(1) 燃空比同为 1.0 的二甲醚和乙醇火焰中间物 种种类相近, 区别在于: 在前者中探测到了甲基乙 基醚, 而在后者中有甲氧基出现, 乙醇火焰反应区域 略宽于二甲醚火焰.

(2) 甲醛是两火焰最主要的 $\mathrm{C}_{1}$ 中间物种, 其摩 尔分数明显高于其他 $\mathrm{C}_{1}$ 物种, 两火焰中甲醛摩尔分 数峰值接近, 乙醇火焰中甲醛存在区域略宽于二甲 醚火焰. 相对于二甲醚火焰, 乙醇火焰中甲基、甲烷 摩尔分数较低, 甲醇的生成反应略滞后于二甲醚火 焰.

(3) 二甲醚火焰趋向于生成 $\mathrm{C}_{1}$ 中间物种, $\mathrm{C}_{2}$ 中 间物种摩尔分数较低, 乙烯、乙醛分别为二甲醚、乙 醇火焰中摩尔分数最高的 $\mathrm{C}_{2}$ 中间物种. 乙醇火焰 中, 包括乙醛、乙烯、乙炔和乙烯酮在内的 $\mathrm{C}_{2}$ 中间物 种摩尔分数明显高于二甲醚火焰.

致谢：本工作得到国家同步辐射实验室资助. 感谢国家同 步辐射实验室燃烧与火焰实验站所有老师、同学对实验工作 的支持和指导.

\section{References}

1 Semelsberger, T. A.; Borup, R. L.; Greene, H. L. J. Power Sources, 2006, 156(2): 497

2 Jiang, D. M.; Huang, Z. H. Combustion science of internalcombustion engine substitute fuel. $\mathrm{Xi}^{\prime}$ an: $\mathrm{Xi}^{\prime}$ an Jiaotong University Press, 2007: 3-12 [蒋德明, 黄佐华. 内燃机替代燃料燃烧学. 
西安: 西安交通大学出版社, 2007: 3-12]

3 Cool, T. A.; Wang, J.; Hansen, N.; Westmoreland, P. R.; Dryer, F. L.; Zhao, Z.; Kazakov, A.; Kasper, T.; Kohse-Höinghaus, K. Proc. Combust. Inst., 2007, 31(1): 285

4 Zhao, Z.; Chaos, M.; Kazakov, A.; Dryer, F. L. Int. J. Chem. Kinet., 2008, 40(1): 1

5 Kasper, T. S.; Oßwald, P.; Kamphus, M.; Kohse-Höinghaus, K. Combust. Flame, 2007, 150(3): 220

6 Kohse-Höinghaus, K.; Oßwald, P.; Struckmeier, U.; Kasper, T.; Hansen, N.; Taatjes, C. A.; Wang, J.; Cool, T. A.; Gon, S.; Westmoreland, P. R. Proc. Combust. Inst., 2007, 31(1): 1119

7 Wang, J.; Struckmeier, U.; Yang, B.; Cool, T. A.; Osswald, P.; Kohse-Hoeinghaus, K.; Kasper, T.; Hansen, N.; Westmoreland, P. R. J. Phys. Chem. A, 2008, 112(39): 9255

8 Song, K. H.; Nag, P.; Litzinger, T. A.; Haworth, D. C. Combust. Flame, 2003, 135(3): 341

9 Nick, M. M. Int. J. Chem. Kinet., 1999, 31(3): 183

10 Norton, T. S.; Dryer, F. L. Int. J. Chem. Kinet., 1992, 24(4): 319

11 Zhu, C. J.; Li, J.; Gao, Y.; Yang, T. Transactions of the Chinese Society for Agricultural Machinery, 2008, 39(9): 6 ～朱昌吉, 李 君, 高 莹, 杨 涛. 农业机械学报, 2008, 39(9): 6]

12 Zhang, J. J.; Qiao, X. Q.; Guan, B.; Wang, Z.; Xiao, G. F.; Huang, Z. Transactions of CSICE, 2008, 26(3): 193 [张俊军, 乔信起, 管 斌, 王 真, 肖广飞, 黄 震. 内燃机学报, 2008, 26(3): 193]

13 Chang, G. F.; Guo, Y. N.; Zhang, J. P.; Wang, Y. K.; Liu, X. J.;
Xu, S. C. Journal of Combustion Science and Technology, 2008, 14(4): 305 [常国峰, 郭英男, 张纪鹏, 王有坤, 刘巽俊, 许思传. 燃烧科学与技术, 2008, 14(4): 305]

14 Yao, C. D.; Liu, X. Y.; Li, J.; Wang, J.; Tian, Z. Y.; Li, Y. Y. Transactions of CSICE, 2007, 25(5): 390 [姚春德, 刘士钰, 李 菁, 王 晶, 田振玉, 李玉阳. 内燃机学报, 2007, 25(5): 390]

15 Xing, Y.; Yao, M. F.; Zhang, F. G.; Zheng, Z. Q. Transactions of CSICE, 2007, 25(1): 24 [邢 元, 尧命发, 张福根, 郑尊清. 内燃机学报, 2007, 25(1): 24]

16 Zhang, B.; Fu, W. B.; Gong, J. S. Fuel, 2006, 85(5-6): 778

17 Curran, H. J.; Pitz, W. J.; Westbrook, C. K.; Dagaut, P.; Boettner, J. C.; Cathonnet, M. Int. J. Chem. Kinet., 1998, 30(3): 229

18 Fischer, S. L.; Dryer, F. L.; Curran, H. J. Int. J. Chem. Kinet., 2000, 32(12): 713

19 Rosado-Reyes, C. M.; Francisco, J. S.; Szente, J. J.; Maricq, M. M.; Ostergaard, L. F. J. Phys. Chem. A, 2005, 109(48): 10940

20 Qi, F.; Yang, R.; Yang, B.; Huang, C. Q.; Wei, L. X.; Wang, J.; Sheng, L. S.; Zhang, Y. W. Rev. Sci. Instrum., 2006, 77(8): 084101

21 Cool, T. A.; Nakajima, K.; Taatjes, C. A.; McIlroy, A.; Westmoreland, P. R.; Law, M. E.; Morel, A. Proc. Combust. Inst., 2005, 30(1): 1681

22 Cool, T. A.; Nakajima, K.; Mostefaoui, T. A.; Qi, F.; McIlroy, A.; Westmoreland, P. R.; Law, M. E.; Poisson, L.; Peterka, D. S.; Ahmed, M. J. Chem. Phys., 2003, 119(16): 8356 